\title{
Muon 'Depth - Intensity' Relation Measured by LVD Underground Experiment and Cosmic-Ray Muon Spectrum at Sea Level
}

\section{The LVD Collaboration}

M.Aglietta ${ }^{16}$, B.Alpat ${ }^{13}$, E.D.Alyea ${ }^{7}$, P.Antonioli ${ }^{1}$, G.Badino ${ }^{16}$, G.Bari ${ }^{1}$, M.Basile ${ }^{1}$, V.S.Berezinsky ${ }^{10}$, F.Bersani ${ }^{1}$, M.Bertaina ${ }^{16}$, R.Bertoni ${ }^{16}$, G.Bonoli ${ }^{1}$, A.Bosco ${ }^{2}$, G.Bruni ${ }^{1}$, G.Cara Romeo ${ }^{1}$, C.Castagnoli ${ }^{16}$, A.Castellina ${ }^{16}$, A.Chiavassa $^{16}$, J.A.Chinellato ${ }^{3}$, L.Cifarelli ${ }^{1, \dagger}$, F.Cindolo ${ }^{1}$, G.Conforto ${ }^{17}$, A.Contin ${ }^{1}$, V.L.Dadykin ${ }^{10}$, A.De Silva ${ }^{2}$, M.Deutsch $^{8}$, P.Dominici ${ }^{17}$, L.G.Dos Santos ${ }^{3}$, L.Emaldi ${ }^{1}$, R.I.Enikeev ${ }^{10}$, F.L.Fabbri ${ }^{4}$, W.Fulgione ${ }^{16}$, P.Galeotti ${ }^{16}$, C.Ghetti ${ }^{1}$, P.Ghia ${ }^{16}$, P.Giusti ${ }^{1}$, R.Granella ${ }^{16}$, F.Grianti ${ }^{1}$, G.Guidi ${ }^{17}$, E.S.Hafen ${ }^{8}$, P.Haridas ${ }^{8}$, G.Iacobucci ${ }^{1}$, N.Inoue $^{14}$, E.Kemp ${ }^{3}$, F.F.Khalchukov ${ }^{10}$, E.V.Korolkova ${ }^{10}$, P.V.Korchaguin ${ }^{10}$, V.B.Korchaguin ${ }^{10}$,

V.A.Kudryavtsev ${ }^{10, \ddagger}$, K.Lau $^{6}$, M.Luvisetto ${ }^{1}$, G.Maccarone ${ }^{4}$, A.S.Malguin ${ }^{10}$, R.Mantovani ${ }^{17}$, T.Massam ${ }^{1}$, B.Mayes ${ }^{6}$, A.Megna ${ }^{17}$, C.Melagrana ${ }^{16}$, N.Mengotti Silva ${ }^{3}$, C.Morello ${ }^{16}$, J.Moromisato ${ }^{9}$, R.Nania ${ }^{1}$, G.Navarra ${ }^{16}$, L.Panaro ${ }^{16}$, L.Periale $^{16}$, A.Pesci ${ }^{1}$, P.Picchi ${ }^{16}$, L.Pinsky ${ }^{6}$, I.A.Pless ${ }^{8}$, J.Pyrlik ${ }^{6}$, V.G.Ryasny ${ }^{10}$, O.G.Ryazhskaya ${ }^{10}$, O.Saavedra ${ }^{16}$, K.Saitoh $^{15}$, S.Santini ${ }^{17}$, G.Sartorelli ${ }^{1}$, M.Selvi ${ }^{1}$, N.Taborgna ${ }^{5}$, V.P.Talochkin ${ }^{10}$, J.Tang ${ }^{8}$, G.C.Trinchero ${ }^{16}$, S.Tsuji ${ }^{11}$, A.Turtelli ${ }^{3}$, I.Uman ${ }^{13}$, P.Vallania ${ }^{16}$, G. Van Buren ${ }^{8}$, S.Vernetto ${ }^{16}$, F.Vetrano ${ }^{17}$, C.Vigorito ${ }^{16}$, E. von Goeler ${ }^{9}$, L.Votano $^{4}$, T.Wada ${ }^{11}$, R.Weinstein ${ }^{6}$, M.Widgoff ${ }^{2}$, V.F.Yakushev ${ }^{10}$, I.Yamamoto ${ }^{12}$, G.T.Zatsepin ${ }^{10}$, A.Zichichi ${ }^{1}$

${ }^{1}$ University of Bologna and INFN-Bologna, Italy

${ }^{2}$ Brown University, Providence, USA

${ }^{3}$ University of Campinas, Campinas, Brazil

${ }^{4}$ INFN-LNF, Frascati, Italy

${ }^{5}$ INFN-LNGS, Assergi, Italy

${ }^{6}$ University of Houston, Houston, USA

${ }^{7}$ Indiana University, Bloomington, USA

${ }^{8}$ Massachusetts Institute of Technology, Cambridge, USA

${ }^{9}$ Northeastern University, Boston, USA

${ }^{10}$ Institute for Nuclear Research, Russian Academy of Sciences, Moscow, Russia

${ }^{11}$ Okayama University, Okayama, Japan

${ }^{12}$ Okayama University of Science, Okayama, Japan

${ }^{13}$ University of Perugia and INFN-Perugia, Italy

${ }^{14}$ Saitama University, Saitama, Japan

${ }^{15}$ Ashikaga Institute of Technology, Ashikaga, Japan

${ }^{16}$ Institute of Cosmo-Geophysics, CNR, Torino, University of Torino and INFN-Torino, Italy

${ }^{17}$ University of Urbino and INFN-Firenze, Italy

${ }^{\dagger}$ now at University of Salerno and INFN-Salerno, Italy

${ }^{\ddagger}$ now at University of Sheffield, United Kingdom

\begin{abstract}
We present the analysis of the muon events with all muon multiplicities collected during 21804 hours of operation of the first LVD tower. The measured angular distribution of muon intensity has been converted to the 'depth - vertical intensity' relation in the depth range from 3 to $12 \mathrm{~km}$ w.e.. The analysis of this relation allowed to derive the power index, $\gamma$, of the primary all-nucleon spectrum: $\gamma=2.78 \pm 0.05$. The 'depth - vertical intensity' relation has been converted to standard rock and the comparison with the data of other experiments has been done. We present also the derived vertical muon spectrum at sea level.
\end{abstract}

PACS numbers: 13.85.T, 96.40.T

\section{INTRODUCTION}

During the last 30 years the cosmic-ray muon energy spectrum has been studied in many experiments using different methods. These methods can be combined into 3 groups: i) the direct measurements of the muon energy spectrum at the sea level using magnetic spectrometers 113 . (the spectrum up to about $10 \mathrm{TeV}$ was measured at the zenith angles near horizon); ii) the measurements of the energy spectrum of cascades produced by muons at shallow depth [4]; iii) the measurement of the depth-intensity curve deep underground 812,33$]$.

Since the spectrum of primary cosmic-ray nucleons has the power-law form with the power index $\gamma$, the spectrum of $\pi-$ and $K$ - mesons produced by primaries should have also the power-law form with the power index $\gamma_{\pi, K}$. If 
the scaling hypothesis is valid in the fragmentation region at high energies, the value of $\gamma_{\pi, K}$ is approximately equal to $\gamma$. The muon spectrum at the sea level has a more complex form due to the competition between the interaction and decay of their parents. Moreover, the flux of muons produced by pions and kaons strongly depends on the zenith angle because the interaction path length of mesons in the atmosphere varies with the zenith angle $\theta$. The muon spectrum follows the power-law dependence only at high energies $E_{\mu}>>E_{\pi}^{c r}, E_{K}^{c r}$, where $E_{\pi}^{c r}$ and $E_{K}^{c r}$ are the critical energies of pions and kaons in the atmosphere. For $\theta=0$ (vertical) this condition is quite satisfied at $E_{\mu}>2 \mathrm{TeV}$. The power index of the muon spectrum, $\gamma_{\mu}$, in this case is more by 1 than the value of $\gamma_{\pi, K}$.

Despite the numerous experiments which studied the muon spectrum, there are the discrepancies in the published results (values of $\gamma, \gamma_{\pi, K}$ or $\gamma_{\mu}$ ). Most of the experiments carried out in the last 20 years gave the values of $\gamma$ (or $\gamma_{\pi, K}$ ) in the range $2.60-2.80$. But the dispersion of the results is greater than the statistical and systematic errors published by the authors. Even the values of $\gamma$, obtained using one method (for example, by the measurement of the depth-intensity curve) have a large dispersion. Such discrepancy can be due to either the difference between the data themselves, or between the calculated muon intensities used to fit the data, or both.

The muon intensities deep underground have been calculated by many authors using different methods (MonteCarlo simulation of the muon transport, numerical solution of the kinetic equations etc., see, for example, [13 200). However, the discrepancy between the muon survival probabilities and, hence, between the muon intensities, calculated by different authors, is quite large and can result in the significant discrepancy between the final values of $\gamma$.

The LVD (Large Volume Detector), located underground, can measure the atmospheric muon intensities from 3000 $\mathrm{hg} / \mathrm{cm}^{2}$ to $12000 \mathrm{hg} / \mathrm{cm}^{2}$ and above (which correspond to the muon energies at the sea level from $1.5 \mathrm{TeV}$ to 40 $\mathrm{TeV})$ at the zenith angles from $0^{\circ}$ to $90^{\circ}$. This allows us to study the muon spectrum and their characteristics at the energies 1.5-40 TeV (which correspond to the energies of primaries of about 10-400 TeV).

In a previous paper 12] we have presented our measurement of the muon depth-intensity curve and the evaluation of the power index of the meson spectrum in the atmosphere using the depth-intensity relation for single muon events. The muon survival probabilities, used to obtain the value of $\gamma_{\pi, K}$ in [12], have been presented in [21]. They have been calculated using the muon interaction cross-sections from 222 24]. After the publication of these results, new calculation of the cross-section of muon bremsstrahlung and of the corrections to the knock-on electron production cross-section have been done [25]. In the present analysis we have taken into account the corrections proposed in [25] and we have estimated the uncertainties of $\gamma\left(\gamma\right.$ is assumed to be equal to $\left.\gamma_{\pi, K}\right)$ due to the uncertainties of the cross-sections used to simulate the muon transport through the rock. The analysis is based on an increased statistics comparing with the previous publications and refers to events with all muon multiplicities.

In Section 2 the detector and the procedure of data processing together with the conversion of the muon intensity to vertical are briefly described. In Section 3 the results of the analysis of the 'depth - vertical muon intensity' distribution $\left(I_{\mu}(x)\right)$ are presented. In Section 4 the 'depth - vertical intensity' relation in standard rock is compared with the data of other underground experiments. In Section 5 we present the derived muon spectrum at sea level. Section 6 contains the conclusions.

\section{LVD AND DATA PROCESSING}

The LVD (Large Volume Detector) is located in the underground Gran Sasso Laboratory at a minimal depth of about $3000 \mathrm{hg} / \mathrm{cm}^{2}$. The LVD will consist of 5 towers. The 1 st tower is running since June, 1992, and the 2nd one since June, 1994. The data presented here were collected with the 1st LVD tower during 21804 hours of live time.

The 1st LVD tower contains 38 identical modules [28]. Each module consists of 8 scintillation counters and 4 layers of limited streamer tubes (tracking detector) attached to the bottom and to one vertical side of the supporting structure. A detailed description of the detector was given in [28]. One LVD tower has the dimensions of $13 \times 6.3 \times 12$ $\mathrm{m}^{3}$.

The LVD measures the atmospheric muon intensities from $3000 \mathrm{hg} / \mathrm{cm}^{2}$ to more than $12000 \mathrm{hg} / \mathrm{cm}^{2}$ (which correspond to the median muon energies at the sea level from $1.5 \mathrm{TeV}$ to $40 \mathrm{TeV}$ ) at the zenith angles from $0^{\circ}$ to $90^{\circ}$ (on the average, the larger depths correspond to higher zenith angles).

We have used in the analysis the muon events with all multiplicities, as well as the sample of single muons. Our basic results have been obtained with all muon sample. This sample contains about 2 millions of reconstructed muon tracks.

The acceptances for each angular bin have been calculated using the simulation of muons passing through LVD taking into account muon interactions with the detector materials and the detector response. The acceptances for both single and multiple muons were assumed to be the same.

As a result of the data processing the angular distribution of the number of detected muons $N_{\mu}(\phi, \cos \theta)$ has been obtained. The angular bin width $1^{\circ} \times 0.01$ has been used. The analysis refers to the angular bins for which the 
efficiency of the muon detection and track reconstruction is greater than 0.03 . We have excluded from the analysis the angular bins with a large variation of depth.

The measured $N_{\mu}(\phi, \cos \theta)$-distribution has been converted to the 'depth - vertical muon intensity' relation, $I_{\mu}(x)$, using the formula:

$$
I_{\mu}\left(x_{m}\right)=\frac{\sum_{i j} N_{\mu}\left(x_{m}\left(\phi_{j}, \cos \theta_{i}\right)\right) \cdot \cos \theta_{i}^{\star \star}}{\sum_{i j}\left(A\left(x_{m}\left(\phi_{j}, \cos \theta_{i}\right)\right) \epsilon\left(x_{m}\left(\phi_{j}, \cos \theta_{i}\right)\right) \cdot \Omega_{i j} \cdot T\right)}
$$

where the summing up has been done over all angular bins $\left(\phi_{j}, \cos \theta_{i}\right)$ contributing to the $\operatorname{depth} x_{m} ; A\left(x_{m}\left(\phi_{j}, \cos \theta_{i}\right)\right)$ is the cross-section of the detector in the plane perpendicular to the muon track at the angles $\left(\phi_{j}\right.$, cos $\left.\theta_{i}\right)$; $\epsilon\left(x_{m}\left(\phi_{j}, \cos \theta_{i}\right)\right)$ is the efficiency of muon detection and reconstruction; $\Omega_{i j}$ is the solid angle for the angular bin; $T$ is the live time; and $\cos \theta_{i}^{\star \star}=I_{\mu}^{c}\left(x_{m}, \cos \theta=1\right) / I_{\mu}^{c}\left(x_{m}, \cos \theta_{i}\right)$ is the ratio of predicted muon intensity at $\cos \theta=1$ to that at $\cos \theta_{i}$. To obtain the values of $\cos \theta_{i}^{\star \star}$ the eq. (4) (see below) with the previously estimated parameters of the muon spectrum at sea level 12,21] and the calculated survival probabilities have been used. Actually, the values of $\cos \theta_{i}^{\star \star}$ do not depend much on the parameters of the muon spectrum at sea level (normalization factor $A$ and power index $\gamma$ ) for any reasonable values of $\gamma$. The factor $\cos \theta_{i}^{\star \star}$ is different from the simple $\cos \theta-$ law used to $\operatorname{convert}$ the muon intensities to vertical in 12 .

In the calculations of $\cos \theta_{i}^{\star \star}$ the contribution of the prompt muons from charmed particle decay has been neglected. As it is shown in 26, 27] the ratio of prompt muons to pions according to LVD data does not exceed $2 \cdot 10^{-3}$ at $95 \%$ confidence level. This means that at median depths (no more than 6-7 km w.e.) the fraction of prompt muons with respect to conventional muons does not exceed $10 \%$ at vertical. And there is also a little probability that this fraction is more than $10 \%$ at large depths $(8-10 \mathrm{~km}$ w.e. $)$.

For depth - intensity relation the bin width of $200 \mathrm{~m}$ w.e. has been chosen. For depths more than $9 \mathrm{~km}$ w.e. the bin width was increased to $500 \mathrm{~m}$ w.e. to increase the statistics for each bin. The conversion of muon intensity to the middle points of each depth bin has been done using the formula:

$$
I_{\mu}^{m}\left(x_{i}\right)=I_{\mu}^{m}\left(x_{m}\right) \frac{I_{\mu}^{c}\left(x_{i}\right)}{I_{\mu}^{c}\left(x_{m}\right)}
$$

where $I_{\mu}^{m}\left(x_{m}\right)$ and $I_{\mu}^{c}\left(x_{m}\right)$ are the measured and calculated muon intensities at the weighted average depth $x_{m}$ which corresponds to the depth bin with the middle value of $x_{i} ; I_{\mu}^{m}\left(x_{i}\right)$ and $I_{\mu}^{c}\left(x_{i}\right)$ are the derived and calculated muon intensities at the depth $x_{i}$ which is the middle point of the depth bin. The values of $x_{m}$ have been obtained by averaging the depths for all angular bins contributing to the given depth bin with a weight equal to the detected number of muons. To calculate the muon intensities at $x_{m}$ and $x_{i}$ we have used again eq. (4) with the previously estimated parameters of muon spectrum [12,21] and the simulated muon survival probabilities.

Since the width of depth bins is quite small (200 m w.e. for depth bins with high statistics) and the number of angular bins contributing to each depth bin is quite large (several hundreds), the conversion factor does not exceed $10 \%$.

\section{III. 'DEPTH - VERTICAL INTENSITY' RELATION IN GRAN SASSO ROCK}

'Depth - vertical muon intensity' relation derived as it is described in the previous section has been fitted with the calculated distributions with 2 free parameters of the muon spectrum at sea level: normalization constant, $A$, and the power index of the primary all-nucleon spectrum, $\gamma$. To calculate the muon intensities underground we have used the formula:

$$
I_{\mu}(x, \cos \theta)=\int_{0}^{\infty} P\left(E_{\mu 0}, x\right) \frac{d I_{\mu 0}\left(E_{\mu 0}, \cos \theta\right)}{d E_{\mu 0}} d E_{\mu 0}
$$

where $P\left(E_{\mu 0}, x\right)$ is the survival probability of muon with an initial energy $E_{\mu 0}$ at sea level to reach the depth $x$; and $\frac{d I_{\mu 0}\left(E_{\mu 0}, \cos \theta\right)}{d E_{\mu 0}}$ is the muon spectrum at sea level at zenith angle $\theta$. This spectrum has been taken according to 29]:

$$
\begin{aligned}
\frac{d I_{\mu 0}\left(E_{\mu 0}, \cos \theta\right)}{d E_{\mu 0}} & =A \cdot 0.14 \cdot E_{\mu 0}^{-\gamma} \\
& \times\left(\frac{1}{1+\frac{1.1 E_{\mu 0} \cos \theta^{\star}}{115 G e V}}+\frac{0.054}{1+\frac{1.1 E_{\mu 0} \cos \theta^{\star}}{850 G e V}}\right)
\end{aligned}
$$


where the values of $\cos \theta$ have been substituted by $\cos \theta^{\star}$ which have been taken from [30]. In [30] $\cos \theta^{\star}=$ $E_{\pi, K}^{c r}(\cos \theta=1) / E_{\pi, K}^{c r}(\cos \theta)$, where $E_{\pi, K}^{c r}$ are the critical energies of pions and kaons. This formula has been obtained under a simple assumption of scaling in the high-energy hadron-nucleus interactions. Under this assumption the power index of primary spectrum, $\gamma$, is equal to that of meson (pion + kaon) spectrum, $\gamma_{\pi, K}$. To fit the 'depth vertical intensity' relation measured by $L V D$ we have put $\cos \theta=\cos \theta^{\star}=1$, however, to convert the muon intensity to vertical (as it was described in the previous section) we have used the values of $\cos \theta^{\star}$ from [30].

We have used the muon survival probabilities $P\left(E_{\mu 0}, x\right)$ calculated with the muon cross-sections from [23 25] and with the account of stochasticity of all processes of muon interaction with matter. They differ from those presented in 21] and used also in [12] (see also references therein) because of the new corrections for muon bremsstrahlung and knock-on electron production cross-sections proposed in [25] (see also the discussion in [31]).

The measured 'depth - intensity' curve has been fitted with the calculated function (see eq. (3)) with two free parameters: additional normalization constant, $A$, and the power index of primary all-nucleon spectrum, $\gamma$. As a result of the fitting procedure the following values of the free parameters have been obtained: $A=1.95 \pm 0.31$, $\gamma=2.78 \pm 0.02$. These values are in good agreement with the results of the analysis of the depth - angular distributions [26,27. We note that the energy in eq. (勾) is expressed in $\mathrm{GeV}$ and the intensity is expressed in $\mathrm{cm}^{-2} \mathrm{~s}^{-1} \mathrm{sr}^{-1}$. The errors of the parameters include both statistical and systematic uncertainties. The latter one takes into account possible uncertainties in the depth, rock composition, density etc., but does not take into account the uncertainty in the cross-sections used to simulate the muon transport through the rock. If we add the uncertainty in the muon interaction cross-sections, the error of $\gamma$ will increase from 0.02 to 0.05 and the error of $A$ to 1.0 (for the discussion about the uncertainty due to different cross-sections see [31]). Similar analysis performed for single muons reveals almost the same value of power index while the absolute intensity is $10 \%$ smaller: $A=1.65 \pm 0.30, \gamma=2.77 \pm 0.02$. We note that the estimates of the parameters $A$ and $\gamma$ are strongly correlated. The larger the value of $\gamma$ is, the larger the normalization factor $A$ should be. The 'depth - vertical muon intesity' relation is shown in Figure 1 for all muon sample together with the best fit. The muon intensities are presented also in Table (column 2).

If the formula from [32] is used for the muon spectrum at sea level instead of eq. (4i), the best fit values of $\gamma$ will be decreased by 0.04-0.05 and will be in agreement with the previously published values for single muons [12,21] analysed using the formula from 32 .

The value of $\gamma$ obtained with LVD data is in reasonable agreement with the results of many other surface and underground experiments (see, for example, [2, 4, 6, $6[10,11]$ ).

\section{IV. 'DEPTH - VERTICAL MUON INTENSITY' RELATION IN STANDARD ROCK}

The simulations carried out for Gran Sasso and standard rocks allow us to obtain the formula for the conversion of the depth in Gran Sasso rock, $x_{g s}$, to that in standard rock, $x_{s t}$. This was done by comparing the values of $x_{s t}$ and $x_{g s}$ for the same muon intensity: $I_{\mu}\left(x_{s t}\right)=I_{\mu}\left(x_{g s}\right)$. The muon intensities have been calculated with the value of $\gamma$ which fit well the LVD data. The depth in standard rock can be evaluated from the depth in Gran Sasso rock using the formula:

$$
x_{s t}=-9.344+1.0063 x_{g s}+1.7835 \cdot 10^{-6} x_{g s}^{2}-5.7146 \cdot 10^{-11} x_{g s}^{3},
$$

where the depth is measured in $\mathrm{hg} / \mathrm{cm}^{2}$. This formula is valid for depth range 1-12 $\mathrm{km}$ w.e.. It has been used to convert the depth- muon intensity relation measured in Gran Sasso rock to that in standard rock to allow the comparison with the data of other experiments.

The 'depth - vertical intensity' relation in the standard rock for all muon sample is presented in Figure 2. It can be fitted with a three parameter function:

$$
I_{\mu}(x)=A\left(\frac{x_{0}}{x}\right)^{\alpha} \exp ^{-\frac{x}{x_{0}}},
$$

where $A=(2.15 \pm 0.08) \cdot 10^{-6} \mathrm{~cm}^{-2} \mathrm{~s}^{-1} \mathrm{sr}^{-1}, x_{0}=\left(1155_{-30}^{+60}\right) \mathrm{hg} / \mathrm{cm}^{2}, \alpha=1.93_{-0.12}^{+0.20}$.

The best fit function is also shown in Figure 2 by solid curve. The LVD data converted to the standard rock agree quite well with the best fit functions for the data of MACRO [11] (dashed curve in Figure 2) and Frejus [33] (dotted curve which almost coincide with the solid curve) underground experiments, despite the difference in the formulae used for depth conversion. The LVD data do not contradict also to the function which fit the data of NUSEX experiment [10] (dash-dotted curve).

The muon intensities measured by LVD and converted to the standard rock are presented also in Table I (3rd column). 


\section{MUON ENERGY SPECTRUM AT THE SEA LEVEL}

Using eq. (4) with the estimates of the free parameters, the LVD data for Gran Sasso rock can be converted to the vertical muon spectrum at the sea level. The simple normalization procedure has been applied:

$$
I_{\mu 0}^{m}\left(x=0, E_{m 0}\right)=\frac{I_{\mu}^{m}(x) I_{\mu 0}^{c}\left(x=0, E_{m 0}\right)}{I_{\mu}^{c}(x)},
$$

where $I_{\mu}^{m}(x)$ and $I_{\mu}^{c}(x)$ are the measured and calculated vertical muon intensities at the depth $x$ in the Gran Sasso rock, $I_{\mu 0}^{m}\left(x=0, E_{m 0}\right)$ and $I_{\mu 0}^{c}\left(x=0, E_{m 0}\right)$ are the derived and calculated (using eq. (4) ) differential vertical muon intensities at energy $E_{m 0}$ at sea level. The median energies $E_{m 0}(x)$ which determine the muon intensity at the depth $x$ have been calculated using the equation:

$$
\frac{I_{\mu}(x)}{2}=\int_{0}^{E_{m 0}} P\left(E_{\mu 0}, x\right) \cdot \frac{d I_{\mu 0}\left(E_{\mu 0}\right)}{d E_{\mu 0}} \cdot d E_{\mu 0}
$$

where $\frac{d I_{\mu 0}\left(E_{\mu 0}\right)}{d E_{\mu 0}}$ is the muon energy spectrum at sea level. The values of $I_{\mu 0}^{m}\left(x=0, E_{m 0}\right)$ derived from LVD data are presented in Table [1]. The derived (full circles) and calculated (middle solid curve) muon spectra at the sea level are shown in Figure 3 together with the data of MSU [7] (diamonds), ASD [4] (open circles) and the best fit of MACRO 11. (dashed line). Upper and lower solid curves in Figure 3 represent the errors in the parameters and additional $10 \%$ error in the absolute normalization of the muon flux. Muon intensities at sea level derived from LVD data are presented also in Table [1].

\section{CONCLUSIONS}

The angular distribution of muon intensity measured by LVD has been converted to the 'depth - vertical muon intensity' relation. The analysis of this relation in the depth range $3000-12000 \mathrm{hg} / \mathrm{cm}^{2}$ has been done and the parameters of the muon spectrum at sea level have been obtained: $A=1.9 \pm 1.0, \gamma=2.78 \pm 0.05$. The errors include both statistical and systematic errors with the systematic error due to the uncertainty of the muon interaction crosssections dominating. Similar analysis performed for single muon events revealed almost the same value of power index, while the absolute intensity is $10 \%$ smaller. The 'depth - intensity' relation has been converted to the standard rock and fitted with a three-parameter function. This relation agrees well with the data of other underground experiments. Using the measured 'depth - intensity' curve and the estimated parameters of the muon spectrum at sea level we have derived the vertical muon energy spectrum at sea level.

\section{ACKNOWLEDGEMENTS}

We wish to thank the staff of the Gran Sasso Laboratory for their aid and collaboration. This work is supported by the Italian Institute for Nuclear Physics (INFN) and in part by the Italian Ministry of University and ScientificTechnological Research (MURST), the Russian Ministry of Science and Technologies, the Russian Foundation of Basic Research (grant 96-02-19007), the US Department of Energy, the US National Science Foundation, the State of Texas under its TATRP program, and Brown University.

[1] M.G.Thompson et al., Proc. 15th Intern. Cosmic Ray Conf. (Plovdiv) 6 (1977) 21.

[2] O.C.Allkofer et al., Proc. 17th Intern. Cosmic Ray Conf. (Paris) 10 (1981) 321.

[3] S.Matsuno et al., Phys. Rev. D 29 (1984) 1.

[4] F.F.Khalchukov et al., Proc. 19th Intern. Cosmic Ray Conf. (La Jolla) 8 (1985) 12; R.I.Enikeev et al., Sov. J. Nucl. Phys. 47 (1988) 1044.

[5] V.N.Bakatanov et al., Proc. 21st Intern. Cosmic Ray Conf. (Adelaide) 9 (1990) 375.

[6] V.D.Ashitkov et al., Proc. 19th Intern. Cosmic Ray Conf. (La Jolla) 8 (1985) 77. 
[7] N.P.Il'ina et al., Proc. 24st Intern. Cosmic Ray Conf. (Rome) 1 (1995) 524.

[8] M.R.Krishnaswami et al., Proc. 18th Intern. Cosmic Ray Conf. (Bangalore) 11 (1983) 450.

[9] Yu.M. Andreyev, V.I.Gurentsov, and I.M.Kogai, Proc. 20th Intern. Cosmic Ray Conf. (Moscow) 6 (1987) 200.

[10] G.Battistoni et al., Nuovo Cimento 9C (1986) 196.

[11] M.Ambrosio et al. (MACRO Collaboration), Phys. Rev. D 52 (1995) 3793.

[12] M.Aglietta et al. (LVD Collaboration), Astroparticle Phys. 3 (1995) 311.

[13] V.I.Gurentsov, G.T.Zatsepin, and E.D.Mikhalchi, Sov. J. Nucl. Phys. 23 (1976) 1001.

[14] V.I.Gurentsov, Preprint INR, P-0380 (1984) (in Russian).

[15] H.Bilokon et al., Nucl. Instrum. and Meth. in Phys. Res. A303 (1991) 381.

[16] H.Bilokon et al., Preprint LNGS 94/92 (1994).

[17] P.Lipari and T.Stanev, Phys. Rev. D 44 (1991) 3543.

[18] V.A.Kudryavtsev, Preprint INR, P-0529 (1987) (in Russian).

[19] E.V.Bugaev et al., Preprint INR, P-0347 (1984) (in Russian).

[20] A.A.Lagutin, Preprint ASU, 94/1 (1994).

[21] M.Aglietta et al. (LVD Collaboration), Proc. 24th Intern. Cosmic Ray Conf. (Rome) 1 (1995) 557.

[22] L.B.Bezrukov and E.V.Bugaev, Proc. 17th Intern. Cosmic Ray Conf. (Paris) 7 (1981) 102.

[23] L.B.Bezrukov and E.V.Bugaev, Proc. 17th Intern. Cosmic Ray Conf. (Paris) 7 (1981) 90.

[24] R.P.Kokoulin and A.A.Petrukhin, Proc. 12th Intern. Cosmic Ray Conf. (Hobart) 6 (1971) 2436.

[25] S.R.Kelner, R.P.Kokoulin, and A.A.Petrukhin, Physics of Atomic Nuclei 60 (1997) 576.

[26] LVD Collaboration (M.Aglietta et al.), Proc. 25th ICRC (Durban) 6 (1997) 341.

[27] LVD Collaboration (M.Aglietta et al.), to be submitted to Phys. Rev. D.

[28] M.Aglietta et al., Astroparticle Phys. 2 (1994) 103.

[29] T.K.Gaisser, Cosmic Rays and Particle Physics (Cambridge University Press, 1990)

[30] L.V.Volkova, Preprint Lebedev Physical Institute N 72 (1969).

[31] P.Antonioli et al., Astroparticle Phys. 7 (1997) 357.

[32] L.V.Volkova, G.T.Zatsepin, and L.A.Kuzmichev, Sov. J. Nucl. Phys. 29 (1979) 1252.

[33] Ch.Berger et al., Phys. Rev. D 40 (1989) 2163. 


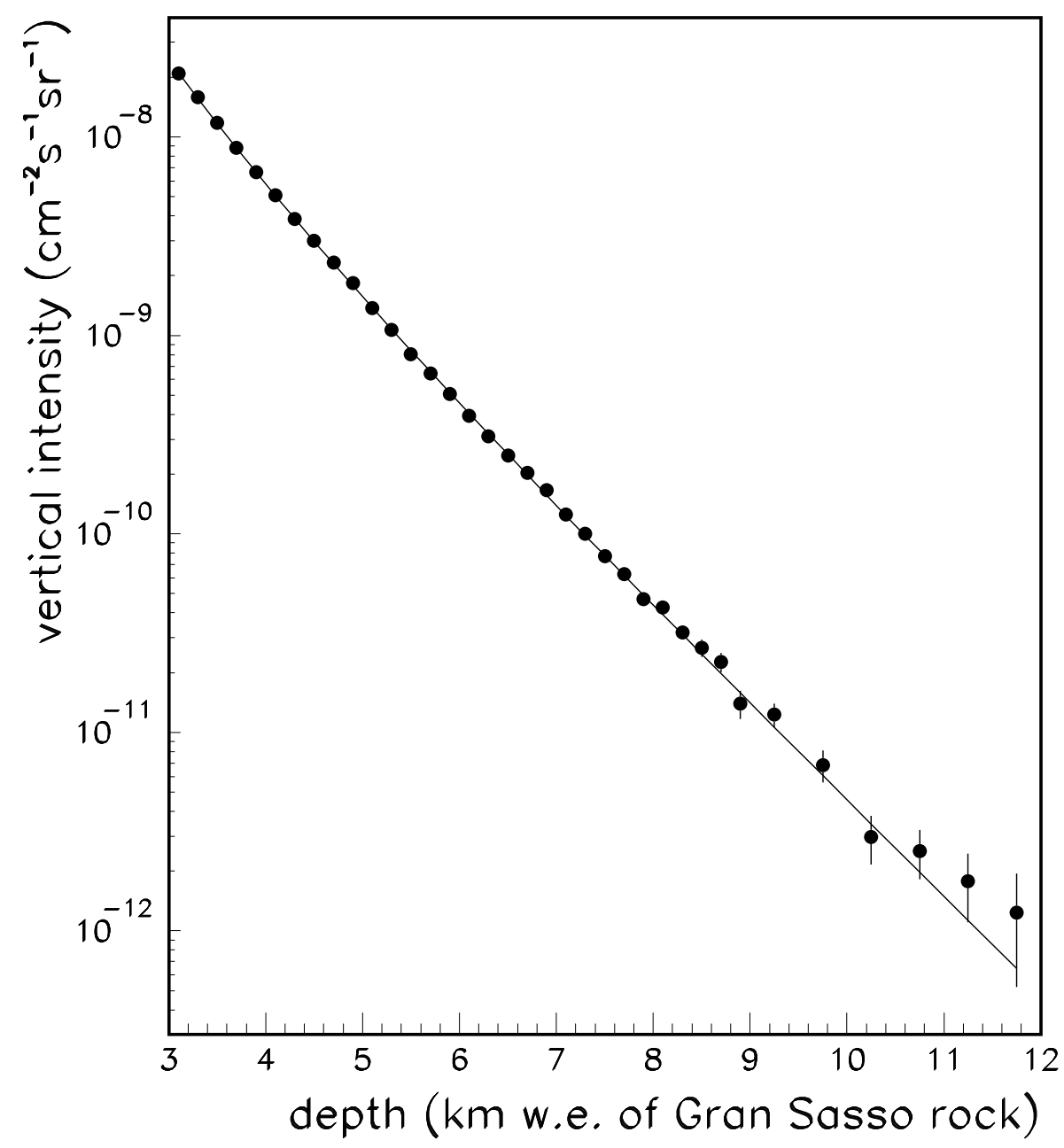

FIG. 1. 'Depth - vertical muon intensity' curve in Gran Sasso rock measured by LVD together with the best fit using eq. (四) with the parameters: $\gamma=2.78$ and $A=1.95$. 


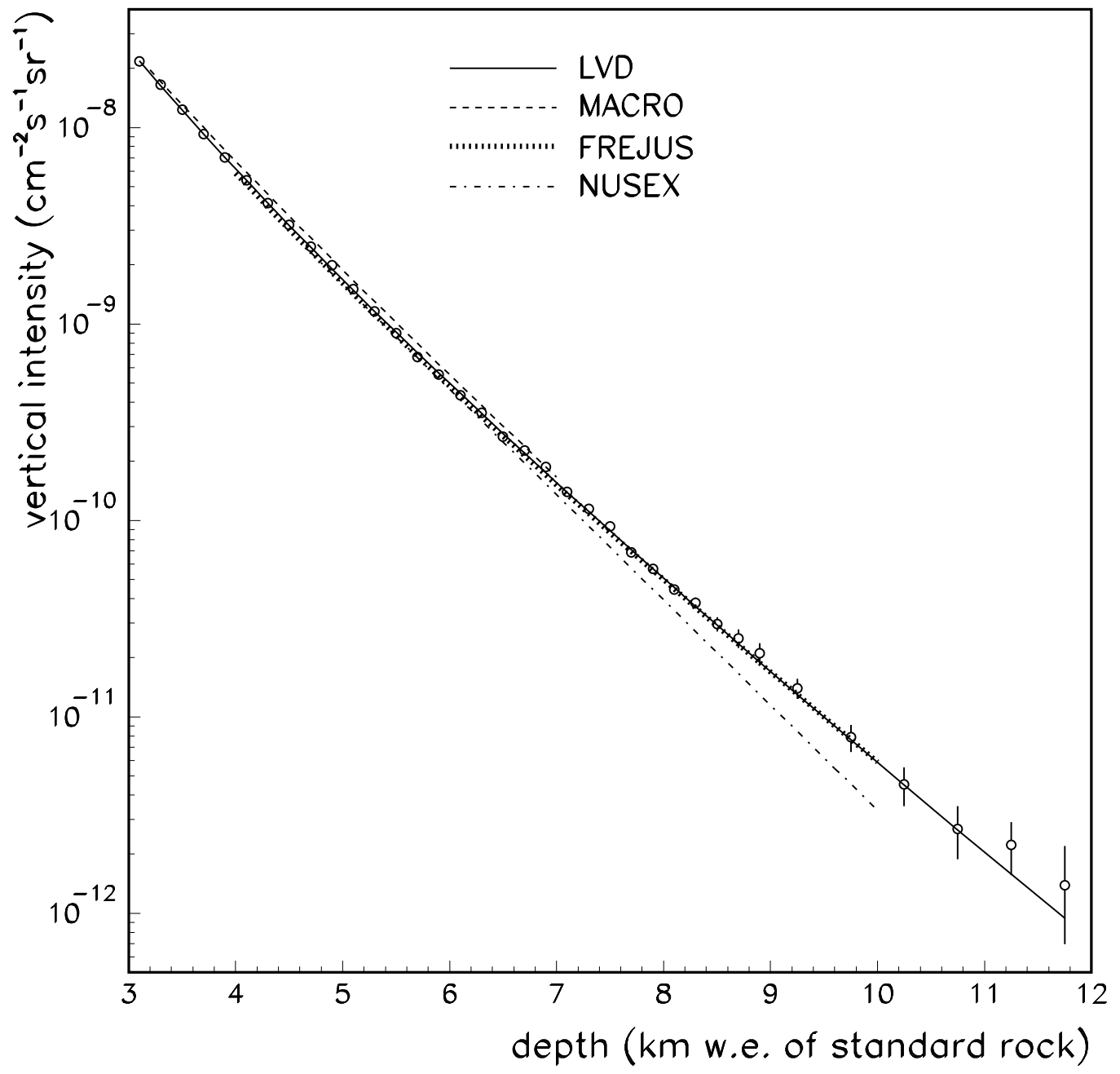

FIG. 2. 'Depth - vertical muon intensity' curve in standard rock. LVD data are presented together with the best fit using three-parameter function (see eq. (6)) and the best fits to the data of other experiments: MACRO [11] (dashed curve), Frejus [33] (dotted curve) and NUSEX [10] (dash-dotted curve). 


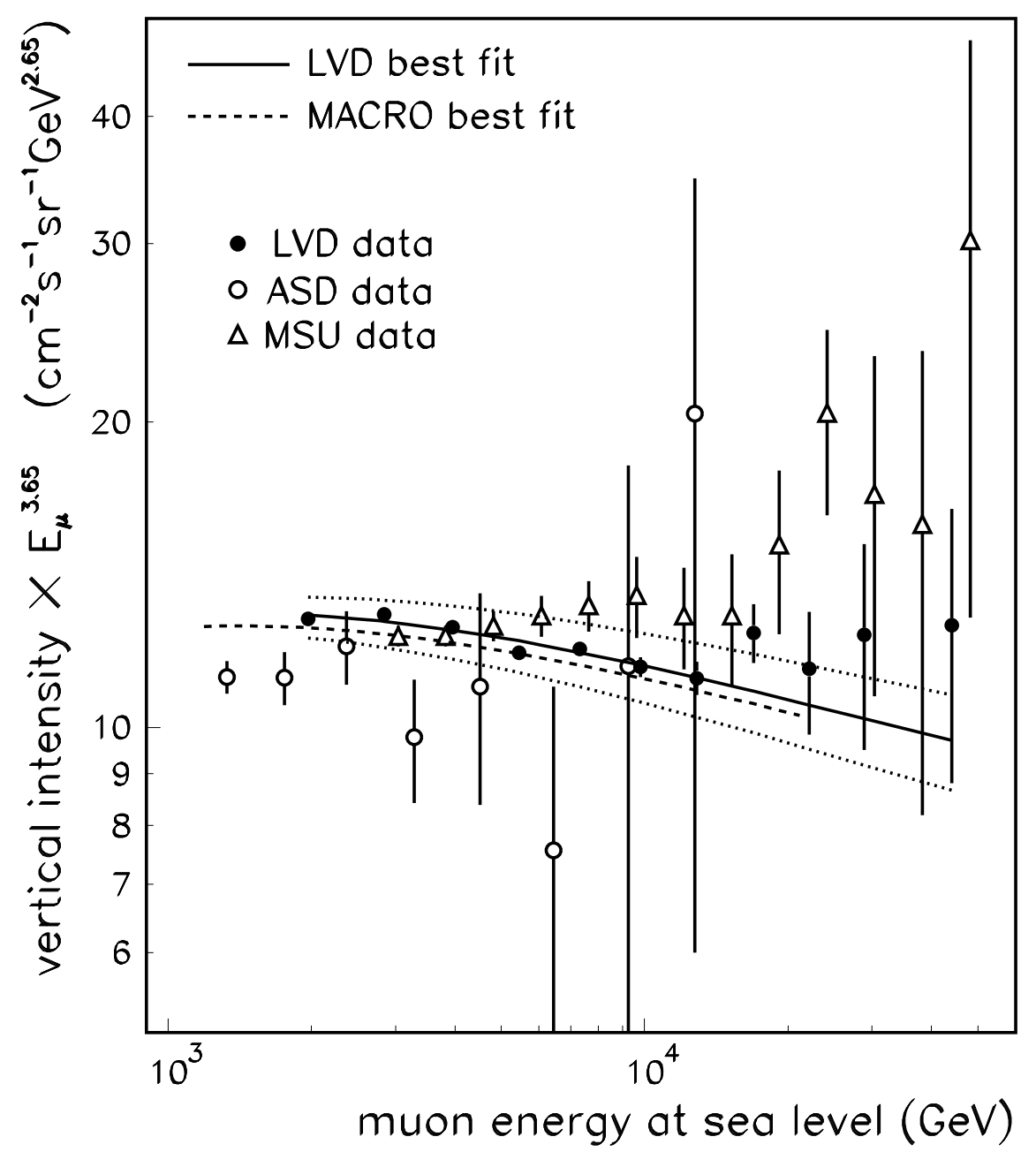

FIG. 3. Vertical muon energy spectra at sea level: • - LVD data; ○ - ASD data [4]; $\diamond-$ MSU data [7]; solid curves - LVD best fit together with the curves representing the errors of the parameters and of the absolute flux normalization; dashed curve - MACRO best fit [11]. 
TABLE I. Vertical muon intensities measured by LVD vs depth in the Gran Sasso and standard rocks. Errors include both statistical and systematic uncertainties.

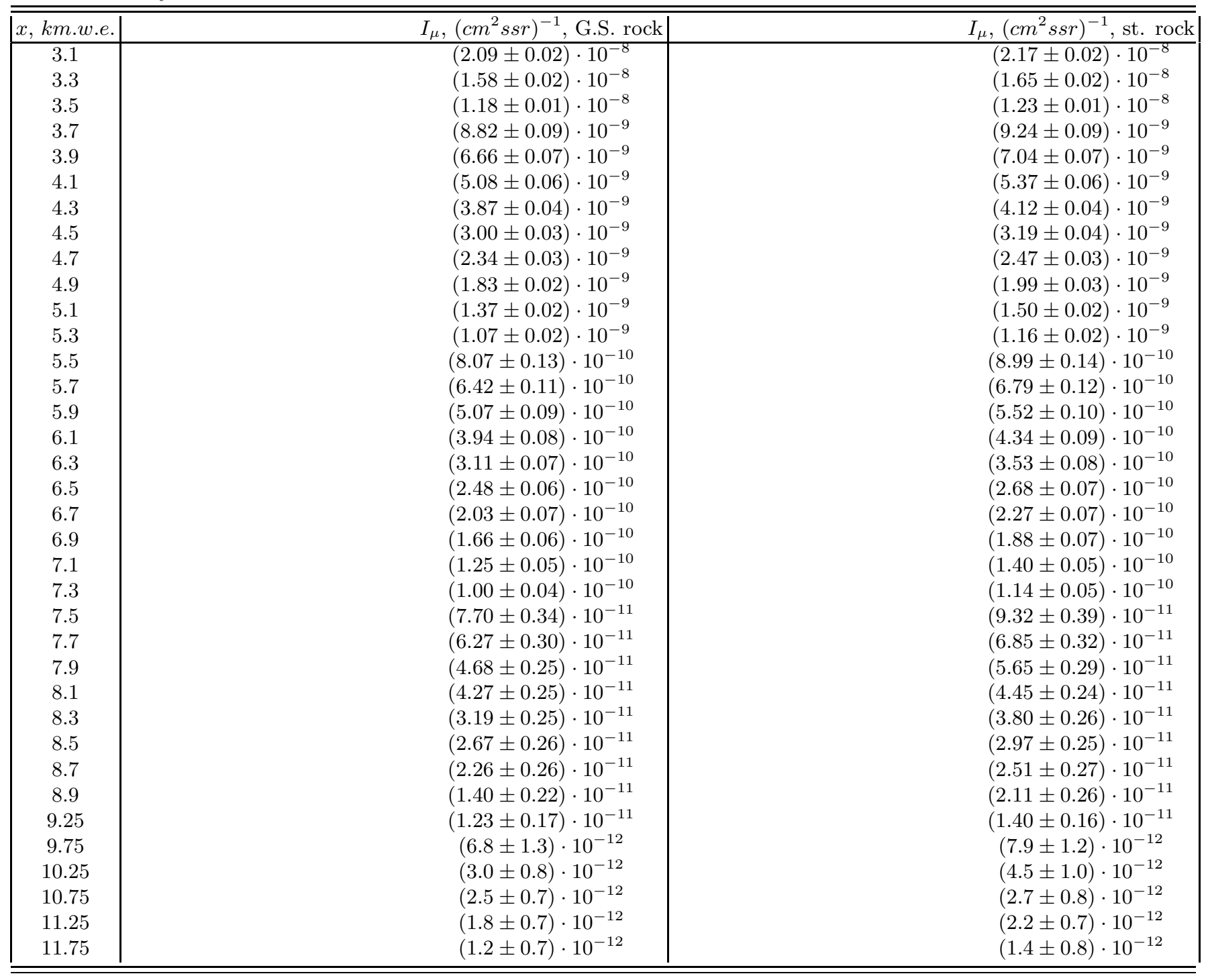


TABLE II. Vertical muon spectrum at sea level derived from LVD data. Errors include both statistical and systematic uncertainties.

\begin{tabular}{|c|c|}
\hline$E_{\mu 0}, \mathrm{GeV}$ & $I_{\mu}, \mathrm{cm}^{-2} \mathrm{~s}^{-1} \mathrm{sr}^{-1} \mathrm{GeV}^{-1}$ \\
\hline 1932 & $(1.30 \pm 0.01) \cdot 10^{-11}$ \\
\hline 2793 & $(3.42 \pm 0.03) \cdot 10^{-12}$ \\
\hline 3909 & $(9.72 \pm 0.11) \cdot 10^{-13}$ \\
\hline 7246 & $(9.73 \pm 0.16) \cdot 10^{-14}$ \\
\hline 9685 & $(3.24 \pm 0.07) \cdot 10^{-14}$ \\
\hline 12770 & $(1.15 \pm 0.04) \cdot 10^{-14}$ \\
\hline 21980 & $(1.62 \pm 0.22) \cdot 10^{-15}$ \\
\hline 28580 & $(6.7 \pm 1.5) \cdot 10^{-16}$ \\
\hline 42660 & $(1.6 \pm 0.5) \cdot 10^{-16}$ \\
\hline
\end{tabular}

